\title{
Validade da Versão em Português da Toronto Alexithymia Scale-TAS em Amostra Clínica
}

\author{
Validity of the Portuguese Version of the Toronto Alexithymia Scale-TAS \\ in a Sample of Inpatients
}

\author{
Elisa Medici Pizão Yoshida* \\ Pontificia Universidade Católica de Campinas, Campinas, Brasil
}

\begin{abstract}
Resumo
Foram estudadas propriedades psicométricas da versão em português da Toronto Alexithymia Scale-TAS, em uma amostra de 294 pacientes internados em hospital geral por diversas patologias. Os resultados mostraram boa consistência interna $(\mathrm{a}=0,72)$, e precisão de teste e reteste $(r=0,72)$. Análise fatorial sugeriu que um modelo de três dimensões pode ser mais adequado para esta população. Os itens do primeiro fator estão associados à habilidade para identificar os sentimentos e distingui-los de sensações corporais. Os itens do segundo fator relacionam-se a um estilo concreto de pensamento. Os itens do terceiro fator relacionam-se à habilidade de expressão de afetos e fantasias (daydreaming). Conclui-se que a alexitimia, conforme avaliada pela versão em português da TAS, é multidimensional não sendo bem representada por um escore global de severidade.

Palavras-chave: Emoção; afeto; auto-relato.
\end{abstract}

\begin{abstract}
Psychometric properties of the Portuguese version of the Toronto Alexithymia Scale-TAS were studied in a sample of 294 inpatients with different pathologies, assisted at a general hospital. Results showed good internal consistency $(\mathrm{a}=0,72)$ and test-retest reliability $(\mathrm{r}=0,72)$. For this population the data were best represented by a three-factor structure. The items on the first factor are associated with the ability to identify one's feelings and to distinguish them from bodily sensations. The items on the second subscale relate to a concrete style of thinking. The items on the third subscale pertain to daydreaming and imagination. In conclusion, alexithymia, as measured by the Portuguese version of the TAS, is multidimensional and not well-represented by a global severity score.

Keywords: Emotion; affect; self-report
\end{abstract}

Ainda que a natureza específica da alexitimia não tenha sido de todo elucidada, desde que proposta nos anos 70 por Sifneos (1972/1977), há um consenso entre os autores de que ela representa um construto multidimensional relativo a um déficit no processamento cognitivo e de regulação das emoções que se tornam indiferenciadas e são pobremente reguladas (Haviland, Shaw, MacMurray \& Cummings, 1988). Como conseqüência, o sujeito tende a responder a situações estressantes e/ou conflituosas através de ações que não são reconhecidas como tendo conteúdo afetivo (Martinez-Sánchez, Ato-García \& OrtizSoria, 2003). Observa-se, ainda, uma reduzida capacidade de fantasiar e um padrão de pensamento voltado para os eventos exteriores, com ênfase nos detalhes. Esta forma de pensar apresenta grande semelhança com o pensamento operatório proposto por Marty e de M'Uzan (1963/ 1994). Isto é, um estilo de pensamento caracterizado por falta de fantasia e de sonhos, que têm relação óbvia com os

\footnotetext{
* Endereço para correspondência: Av. Francisco de Assis Dinis, 227, Parque dos Príncipes, Osasco, SP, 06030-380 Tel.: (11) 3684-1497. E-mail: eyoshida.tln@terra.com.br
}

resíduos diurnos. A atividade mental é concreta e orientada para o presente, sem relação com fantasias inconscientes.

Ao longo do tempo, o conceito de alexitimia foi sendo ampliado para se ajustar às observações que apontavam não se tratar de uma característica exclusiva de pacientes psicossomáticos, como inicialmente se aventara (Sifneos, 1972/1977; Sifneos, Apfel-Savitz \& Frankel, 1977), mas uma característica também freqüente em pacientes com transtornos psíquicos, como quadros de depressão e de adição (alcoolismo e drogas), nos quais aparece como um fator predisponente. A alexitimia tem sido, também, encontrada em parcela da população geral, atingindo cerca de $10 \%$ a $20 \%$ de seus membros, enquanto que entre pacientes psicossomáticos, chega a mais de 50\% dos casos (Pedinielli \& Rouan, 1998).

Para dar conta desta diversidade, algumas classificações de alexitimia foram propostas. A mais conhecida é a que a divide em primária e secundária (Freyberger, 1977; Pedinielli \& Rouan, 1998). A primária corresponderia ao conceito original, associada à etiologia dos transtornos psicossomáticos, nos quais atua limitando a expressão dos afetos e das emoções que se manifestam de forma deslocada 
no sintoma físico, no pensamento concreto e na falta de fantasia. Este tipo de expressão, visto como refratário à psicoterapia, especialmente à de orientação psicanalítica, corresponderia a um traço de personalidade. Por outro lado, a alexitimia secundária encontrar-se-ia mais próxima da noção de estado, devido à sua natureza transitória, e poder ser considerada uma das conseqüências do estresse (Consoli, 1996; Pedinielli \& Rouan, 1998). Teria, portanto, função adaptativa, ainda que em certos casos possa realimentar a resposta de estresse, devido exatamente à dificuldade do sujeito de avaliar adequadamente sua resposta emocional.

De uma outra perspectiva, Bermond (1995, citado por Larsen, Brand, Bermond \& Hijman, 2003) propôs que a alexitimia fosse dividida em Tipo I e Tipo II. O Tipo I seria caracterizado pela ausência da experiência emocional relativa a algo vivenciado, que por sua vez impediria a cognição que acompanha a emoção. Já o Tipo II corresponderia a um déficit seletivo da cognição emocional, com escassa experiência emocional. Esta classificação, baseada nas duas vertentes mais afetadas do processamento das emoções - experiência emocional (Tipo I) ou cognição associada às emoções (Tipo II), - mostrou-se útil para o exame dos modelos neurobiológicos que têm servido de base para a fundamentação e explicação do fenômeno alexitímico, ainda que para nenhum deles haja evidências empíricas suficientes para fundamentar teorias consistentes (Larsen et al., 2003).

Na década de 80, Taylor, Ryan e Bagby já se referiam à dificuldade de operacionalizar alexitimia, apesar da consistência das descrições clínicas feitas por pesquisadores de vários países. No texto clássico de 1985, os autores relacionaram os métodos mais comuns de quantificá-la, ainda que não apresentassem qualidades psicométricas aceitáveis. Eram esses: o Beth Israel Hospital Psychosomatic Questionnaire (BIQ), avaliado pelo entrevistador; a escala de auto-relato Schalling-Sifneos Personality Scale (SSPS) e a escala de alexitimia do MMPI. Como alternativa, desenvolveram a Toronto Alexithyimia Scale-TAS, um instrumento de auto-relato que demonstrou estrutura de quatro fatores interpretáveis "congruentes com o construto de alexitimia descrito por teóricos clínicos” (Taylor, Ryan \& Bagby, 1985, p. 197).

Embora a TAS seja o instrumento de avaliação da alexitimia mais utilizado em pesquisas na atualidade, três outros instrumentos merecem referência: o Vorst Alexithymia Questionnaire (Bermont \& Vorst, 1998 citado por Taylor, Bagby \& Luminet, 2000), de auto-relato; o California Q-set Alexithymia Prototype - CAQ-AP(Haviland, 1998; Haviland $\&$ Reise, 1996a), com duas versões: auto-relato e avaliado pelo observador; e a Observer Alexithymia Scale - OAS (Haviland, Warren \& Riggs, 2000; Haviland, Warren, Riggs \& Gallacher, 2001; Haviland, Warren, Riggs \& Nitch, 2002). Vale destacar ainda, que a OAS foi desenvolvida a partir do CAQ-AP e se encontra em fase de adaptação e validação para o português (Carneiro, 2006).
Toronto Alexithymia Scale - TAS

A versão original da TAS, composta por 26 itens, revelou boas qualidades psicométricas, reproduzidas nas versões adaptadas da escala para diferentes idiomas, tais como: o espanhol (Uruguai) (Rodrigo, Lusiardo \& Normey, 1989), o francês (Loas et al., 1993, citado por Jacob \& Hautekeete, 1998), o finlandês (Kauhanen, Julkunen \& Salonen, 1991), o italiano (Bucca et al., 1993, citado por Bressi et al., 1996), entre outros. Isto é, boa validade de critério (Bagby, Taylor \& Ryan, 1986; Taylor et al., 1988), boa consistência interna (alfas entre 0,75 e 0,79) (Bagby, Taylor \& Atkinson, 1988; Taylor et al., 1985), estabilidade temporal em medidas de teste e reteste de uma semana $(r=0,82)$ e cinco semanas $(r=0,75)$ (Taylor et al.,1985) e estudos de análise fatorial que revelaram quatro fatores consistentes com o modelo teórico. Isto é, F1 reflete a habilidade de identificar e descrever sentimentos e distinguir sentimentos de sensações corporais; F2, o sonhar acordado (daydreaming); F3, a preferência por focalizar eventos externos em vez de experiências internas e F4, a habilidade para comunicar os sentimentos a outras pessoas (Bagby, Taylor, Parker \& Loiselles, 1990).

A versão em português também demonstrou boas qualidades psicométricas em amostra de universitários (Yoshida, 2000). Obteve-se escore médio da amostra feminina $(n=394)$ igual a $63,69(D P=10,01)$; da amostra masculina $(n=187) 62,08(D P=10,19)$ e da amostra total $(n=581), 63,13(D P=10,12)$; coeficientes alfa $0,72(\mathrm{~F}), 0,70$ $(\mathrm{M})$ e $0,71(\mathrm{~T})$, precisão de teste e reteste de uma semana o,72. A análise fatorial exploratória indicou quatro componentes, sendo que o Fator 1 contribuiu com 17,92\% da variância, o Fator 2, com 9,73\% da variância, o Fator 3, com 6,63\%, o Fator 4, com 5,39 \%, e os quatro fatores juntos, $39,67 \%$ da variância. Quanto às variáveis psicológicas que eles representam, foram as mesmas da escala original (Bagby et al., 1990).

Apesar das propriedades psicométricas da TAS-26, ou simplesmente TAS, terem sido mais satisfatórias que os instrumentos que a antecederam, na dimensão que avalia "fantasia reduzida" (fator 3) foi identificada correlação negativa com a que avalia "habilidade de distinguir sentimentos de sensações corporais e emoções" (fator 1) (Bagby et al., 1990). Além disso, itens do fator três apresentaram baixa correlação item-total $(r<0,10)$ com a TAS completa. Estas limitações levaram os autores da escala original a desenvolver uma versão mais reduzida com 23 itens, a (TAS-R) - Revised Toronto Alexithymia Scale e, finalmente, uma versão com 20 itens - a TAS-2O - que avalia três dimensões da alexitimia: dificuldade em identificar sentimentos (F1), dificuldade em descrever sentimentos (F2) e pensamento externamente orientado (F3) (Bagby, Parker, \& Taylor, 1994; Bagby, Taylor \& Parker, 1994).

A TAS-20 foi recentemente adaptada para o português por Wiethaeuper, Balbinotti, Pelisoli e Barbosa (2005), em um estudo sobre suas qualidade psicométricas com estudantes universitários. Seus resultados foram comparados aos obtidos em 12 outros países: Finlândia, França, Bélgica, Alemanha, Coréia do Sul, Tailândia, Noruega, Polônia, 
Portugal, Espanha, Peru e Suécia. O estudo da consistência interna para a escala total $($ alfa $=0,76)$ mostrou-se comparável com os dos estudos dos demais países, nos quais os alfa variaram entre 0,68 e 0,84. Uma análise fatorial confirmatória realizada reproduziu três dimensões equivalentes às da versão original e das diferentes versões com as quais foi comparada.

Embora haja uma tendência crescente na literatura para utilizar a TAS-2O ao invés da TAS, em estudo da estrutura fatorial da TAS-20 com o emprego de uma técnica exploratória que considera a informação total do item, e que corresponde a uma generalização multidimensional do modelo da TRI de dois parâmetros de Lord e Novik (1968, citado por Haviland \& Reise, 1996b), Haviland e Reise (1996b) não obtiveram a reprodução da estrutura fatorial original da escala para nenhuma das duas amostras empregadas: estudantes de medicina $(n=219)$ e pacientes hospitalizados por dependência e abuso de drogas psicoativas $(n=204)$. Na amostra de estudantes, a solução de três fatores, representada pelas dimensões originais, mostrou-se mais adequada, embora não inteiramente. $\mathrm{Na}$ amostra clínica, no entanto, a solução de três fatores foi bastante diferente no que concerne ao elenco de itens que compunham cada um dos fatores. Nela, as dimensões "dificuldade de identificar sentimentos" e "dificuldade de descrever sentimentos" formaram um único fator que mede "déficit de consciência (awareness) emocional". A dimensão "pensamento orientado para o exterior" dividiu-se em dois fatores não correlacionados. Estes resultados levaram os autores a sugerir que a alexitimia, medida pela TAS-20, corresponde a um construto multidimensional que não é bem representado por um escore total de severidade da alexitimia, sendo conveniente a avaliação dos escores parciais relativos a cada um dos fatores.

Bagby, Taylor e Parker (2003) indicam que muitos estudos têm encontrado correlações significantes positivas entre a TAS-26 e a TAS-20, e, inúmeras pesquisas com pacientes de diferentes clínicas médicas - tais como: pacientes com hipertensão (Pregnolatto, 2005), dor crônica (Kosturek, Gregory, Sousou \& Trief, 1998; Millard \& Kinsler, 1992), doenças gástricas (Fukunishi, Kikuchi, Kaji \& Yamasaki, 1997; Medeiros, 2002), distúrbios sexuais (Madioni \& Mammana, 2001; Wise, Osbourne, Strand, Fagan, \& Schmidt, 2002), depressão (Parker, Bagby \& Taylor, 1991; Wise, Mann \& Randell, 1995), entre outras - têm sugerido a utilidade da TAS-26, e portanto, a pertinência da investigação das propriedades psicométricas desta versão com populações específicas. Este é o caso, por exemplo, dos pacientes brasileiros atendidos pelo Sistema Único de Saúde (SUS), que apresentam características culturais diversas das evidenciadas por estudantes universitários e também de pacientes de hospitais gerais de países do primeiro mundo em que a maioria das pesquisas descritas foram realizadas.

A pesquisa teve por finalidade contribuir para o processo de validação da versão em português da Toronto Alexithymia Scale -TAS, iniciado com um estudo de validade de construto com amostra de universitários (Yoshida,
2000). Como a validade de medidas empiricamente desenvolvidas deve ser verificada para os diferentes tipos de população em que o instrumento é utilizado, considerouse relevante analisar a estrutura fatorial desta versão para pacientes de hospital geral brasileiro, importante grupoalvo deste tipo de medida. Avaliou-se ainda, a consistência interna e precisão de teste-reteste.

\section{Método}

\section{Participantes}

A amostra foi constituída por 294 pacientes atendidos pelo SUS, de hospital geral de cidade de médio porte do estado de São Paulo, internados nas seguintes enfermarias: maternidade $(34,6 \%)$, or topedia $(24,9 \%)$, hemodiálise $(17,5 \%)$ e oncologia $(22,1 \%)$. Para a definição do tamanho mínimo da amostra, foi utilizado o critério da "razão itens /sujeitos", entre 5 e 10 sujeitos por item do instrumento. Considerando que a TAS tem 26 itens, a proporção de 11,2 participantes para cada item, foi considerada satisfatória. Este critério contempla ainda o requisito mínimo de 200 sujeitos para a realização de análises fatoriais (Pasquali, 1999). A amostra foi composta por: $61,5 \%$ de mulheres; idades entre 18 e 76 anos $(M=38,56, D P=15,01$ e $M e=$ 37); 3,1\% tinham curso superior, 33,1\% nível médio, 30,7\% cursaram entre a $5^{\mathrm{a}}$ e $8^{\mathrm{a}}$ séries do ensino fundamental e $29,3 \%$ cursaram entre a $1^{\text {a }}$ e $4^{\text {a }}$ séries e $3,8 \%$ eram analfabetos; $22,2 \%$ solteiros, $60,2 \%$ casados/amigados, $12,0 \%$ separados/divorciados e 5,5\% viúvos. O tempo de internação variou entre um e sete dias $(M=3,13, D P=1,73)$. $O$ fato de se tratarem de pacientes atendidos pelo SUS sugere que sejam, em sua maioria, provenientes das classes economicamente menos favorecidas.

\section{Instrumento de Medida}

Versão em Português da Escala de Alexitimia de Toronto TAS de Taylor et al. (1985) (Yoshida, 2000) - instrumento de auto-relato, com 26 itens, idealizado para medir o grau de alexitimia. Os itens são respondidos em escala de tipo Likert de cinco pontos variando entre, 1 (discordo inteiramente) e 5 (concordo plenamente). Os escores totais variam entre 26 e 130, sendo que nas pesquisas internacionais, para escores acima de 74 (inclusive) o sujeito é considerado alexitímico e menores de 62 (inclusive) é considerado não alexitímico (Taylor et al., 1988). Para valores intermediários, (entre 63 e 73) nada se pode afirmar. Não existem ainda pontos de corte estabelecidos para a população brasileira.

\section{Procedimento}

A coleta de dados, realizada junto ao leito do paciente, foi antecedida por um breve rapport, em que se verificava se ele apresentava consciência e condição para avaliar adequadamente a realidade e, especialmente, não padecia de dor que o impedisse de se concentrar na tarefa. Só então eram-lhe explicados os propósitos da pesquisa e se o consultava quanto ao interesse em participar dela. Em caso afirmativo, o Termo de Consentimento Esclarecido era lido 
em voz alta pela aplicadora e, a seguir, assinado pelo participante. A administração da TAS, foi assistida pela aplicadora, que lia os itens ao participante, sem contudo induzir a resposta. Esta adaptação foi necessária em face do baixo nível de escolaridade de parte da amostra. O tempo médio de aplicação da escala foi de 10 minutos. Um subgrupo foi re-testado com um intervalo entre 7 e 10 dias após a primeira testagem. A pesquisa foi aprovada pelo Comitê de Ética para Pesquisas com Seres Humanos da instituição.

\section{Resultados}

\section{Consistência Interna}

O coeficiente alfa para a amostra geral foi 0,72 , um valor intermediário entre o da amostra feminina $(0,73)$ e o da masculina $(0,71)$. Nos três casos, esses resultados foram muito próximos dos encontrados junto aos universitários, respectivamente, 0,71, 0,72 e 0,70 (Yoshida, 2000). Portanto, a diferença de nível educacional das amostras e a diferença no modo aplicação do instrumento (assistida na presente pesquisa e auto-relato nos universitários) parece não ter interferido na consistência interna do ins- trumento. Em ambas, no entanto, os valores ficaram um pouco abaixo da maioria das pesquisas internacionais, em que os alfas variaram entre 0,75 e 0,79 (Bagby et al., 1988; Taylor et al., 1985), fato que pode estar relacionado com questões de ordem cultural ou mesmo semântica da versão em português.

Para verificar a influência de cada item para a consistência geral da escala, foram calculadas, para a amostra geral e de acordo com o sexo, a correlação item-teste que mede o grau de associação do item com a escala completa, incluindo o próprio item; a correlação item-resto, que mede a associação do item com a escala, excluindo o próprio item; e os valores de alfa, excluídos cada um dos itens (Tabela 1). Os itens 1, 7, 11, 13, 15 e 24 apresentam baixa correlação item total com a TAS completa, sendo que os itens 7, 11, 13 e 24 pertencem na versão original ao F3. Este resultado coincide com o observado por Bagby, Parker e Taylor (1994), que também identificaram esta característica em pesquisa com universitários. Os valores de alfa, não chegam a ser muito influenciados pela supressão de cada um dos itens, em nenhuma das três amostras, não resultando portanto a exclusão de nenhum item em melhora significante da consistência geral da escala.

Tabela 1

Correlação Item-Teste, Item-Resto e Alfa da TAS, Amostra Geral e por Sexo

\begin{tabular}{|c|c|c|c|c|c|c|c|c|c|}
\hline \multirow[b]{2}{*}{ Item } & \multicolumn{3}{|c|}{ Geral $(n=294)$} & \multicolumn{3}{|c|}{ Feminina $(n=181)$} & \multicolumn{3}{|c|}{ Masculina $(n=113)$} \\
\hline & Item-teste & Item -resto & alfa & Item & Item-teste & Item -resto & alfa & Item & Item-teste \\
\hline 1 & 0,18 & 0,07 & 0,73 & 1 & 0,18 & 0,07 & 0,73 & 1 & 0,18 \\
\hline 2 & 0,41 & 0,31 & 0,71 & 2 & 0,41 & 0,31 & 0,71 & 2 & 0,41 \\
\hline 3 & 0,45 & 0,34 & 0,71 & 3 & 0,45 & 0,34 & 0,71 & 3 & 0,45 \\
\hline 4 & 0,57 & 0,48 & 0,70 & 4 & 0,57 & 0,48 & 0,70 & 4 & 0,57 \\
\hline 5 & 0,33 & 0,23 & 0,72 & 5 & 0,33 & 0,23 & 0,72 & 5 & 0,33 \\
\hline 6 & 0,25 & 0,15 & 0,72 & 6 & 0,25 & 0,15 & 0,72 & 6 & 0,25 \\
\hline 7 & 0,17 & 0,06 & 0,73 & 7 & 0,17 & 0,06 & 0,73 & 7 & 0,17 \\
\hline 8 & 0,51 & 0,42 & 0,70 & 8 & 0,51 & 0,42 & 0,70 & 8 & 0,51 \\
\hline 9 & 0,23 & 0,11 & 0,72 & 9 & 0,23 & 0,11 & 0,72 & 9 & 0,23 \\
\hline 10 & 0,42 & 0,32 & 0,71 & 10 & 0,42 & 0,32 & 0,71 & 10 & 0,42 \\
\hline 11 & 0,03 & $-0,06$ & 0,73 & 11 & 0,03 & $-0,06$ & 0,73 & 11 & 0,03 \\
\hline 12 & 0,21 & 0,10 & 0,73 & 12 & 0,21 & 0,10 & 0,73 & 12 & 0,21 \\
\hline 13 & 0,11 & O,03 & 0,73 & 13 & 0,11 & O,03 & 0,73 & 13 & 0,11 \\
\hline 14 & 0,49 & 0,40 & 0,70 & 14 & 0,49 & 0,40 & 0,70 & 14 & 0,49 \\
\hline 15 & 0,14 & 0,05 & 0,73 & 15 & 0,14 & 0,05 & 0,73 & 15 & 0,14 \\
\hline 16 & 0,46 & 0,36 & 0,71 & 16 & 0,46 & 0,36 & 0,71 & 16 & 0,46 \\
\hline 17 & 0,55 & 0,47 & 0,70 & 17 & 0,55 & 0,47 & 0,70 & 17 & 0,55 \\
\hline 18 & 0,23 & 0,12 & 0,72 & 18 & 0,23 & 0,12 & 0,72 & 18 & 0,23 \\
\hline 19 & 0,30 & 0,19 & 0,72 & 19 & 0,30 & 0,19 & 0,72 & 19 & 0,30 \\
\hline 20 & 0,57 & 0,49 & 0,70 & 20 & 0,57 & 0,49 & $0,7 \mathrm{O}$ & 20 & 0,57 \\
\hline 21 & 0,14 & 0,06 & 0,73 & 21 & 0,14 & 0,06 & 0,73 & 21 & 0,14 \\
\hline 22 & 0,48 & 0,39 & 0,70 & 22 & 0,48 & 0,39 & 0,70 & 22 & 0,48 \\
\hline 23 & 0,45 & 0,35 & 0,71 & 23 & 0,45 & 0,35 & 0,71 & 23 & 0,45 \\
\hline 24 & 0,14 & 0,04 & 0,73 & 24 & 0,14 & 0,04 & 0,73 & 24 & 0,14 \\
\hline 25 & 0,61 & 0,53 & 0,69 & 25 & 0,61 & 0,53 & 0,69 & 25 & 0,61 \\
\hline 26 & 0,56 & 0,47 & 0,70 & 26 & 0,56 & 0,47 & 0,70 & 26 & 0,56 \\
\hline
\end{tabular}




\section{Precisão de Teste e Reteste}

Quarenta e dois pacientes foram retestados após um período entre sete e dez dias, sendo $71,43 \%$ do sexo masculino. Os coeficientes de correlação de Spearman $\left(\mathrm{r}_{\mathrm{s}}\right)$ para a amostra geral, igual a 0,72 , para a feminina, 0,78 , e para a masculina igual a 0,71 sugerem boa estabilidade temporal da escala. Vale destacar que os universitários apresentaram exatamente o mesmo índice para a amostra total $(0,72, p<0,0001)$, ocasião em que os coeficientes por sexo não chegaram a ser estimados.

\section{Validade de Construto 1}

O escore médio da TAS para a amostra geral (72,81, $D P=9,15)$, corresponde a um valor ligeiramente abaixo do internacionalmente aceito como ponto de corte para definir a presença de alexitimia, igual a 74 (Tabela 2). Não foram observadas diferenças significantes em relação aos escores totais médios das amostras feminina e masculina $[\mathrm{t}(292)=1,46, \mathrm{p}>0,05]$, e nem entre os escores médios das dimensões F2, F3 e F4, cujos valores de $\mathrm{t}(292)$ foram respectivamente, 1,26, 0,57 e 0,24. Apenas em F1 o escore médio foi superior para as mulheres $[\mathrm{t}(292)=2,38$, $p<0,02]$. Por se tratarem de pacientes hospitalizados, é possível que os altos escores estejam refletindo uma expressão secundária da alexitimia à situação estressora, que, conforme indicado, corresponderia a "uma reação estado para os efeitos de doenças físicas sérias, talvez uma defesa contra depressão ou dor, ou ambas" (Campbell, 1996, p. 30).

Comparando estes resultados aos relatados por Maciel e Yoshida (2006), verifica-se que os escores médios foram inferiores aos de alcoolistas em atendimento ambulatorial ( $n=20, M=78,65$ e $D P=7,99)$ e superiores aos do grupo comparativo daquela pesquisa, formado por membros da comunidade ( $n=20, M=69,15$ e $D P=8,68)$. Os escores médios foram também significantemente superiores aos encontrados por Yoshida (2000) na amostra de universitários $(M=63,13$ e $D P=10,12)$, o que sinaliza, portanto, a sensibilidade da escala em discriminar populações com diferentes perfis.

Tabela 2

Resumo dos Escores da TAS, Amostra Geral e por Sexo

\begin{tabular}{|c|c|c|c|c|c|c|c|c|c|c|c|c|}
\hline \multirow[t]{2}{*}{ TAS } & \multicolumn{4}{|c|}{ Geral $(n=294)$} & \multicolumn{4}{|c|}{ Feminina $(n=181)$} & \multicolumn{4}{|c|}{ Masculina $(n=113)$} \\
\hline & $m$ & $D P$ & $\min$. & & $m$ & $D P$ & $\min$. & & $m$ & $D P$ & $\min$. & \\
\hline Total & 72,81 & 9,15 & 42 & Total & 72,81 & 9,15 & 42 & Total & 72,81 & 9,15 & 42 & Total \\
\hline f1 & 32,29 & 6,92 & 11 & f1 & 32,29 & 6,92 & 11 & $\mathrm{f} 1$ & 32,29 & 6,92 & 11 & f1 \\
\hline $\mathrm{f} 2$ & 10,48 & 2,72 & 4 & $\mathrm{f} 2$ & 10,48 & 2,72 & 4 & $\mathrm{f} 2$ & 10,48 & 2,72 & 4 & $\mathrm{f} 2$ \\
\hline f3 & 16,75 & 2,41 & 9 & f3 & 16,75 & 2,41 & 9 & f3 & 16,75 & 2,41 & 9 & f3 \\
\hline $\mathrm{f} 4$ & 13,29 & 3,13 & 5 & $\mathrm{f} 4$ & 13,29 & 3,13 & 5 & $\mathrm{f} 4$ & 13,29 & 3,13 & 5 & $\mathrm{f}_{4}$ \\
\hline
\end{tabular}

\section{Validade de Construto 2}

Para a tomada de decisão quanto ao número de fatores a serem extraídos na análise fatorial, foram considerados os seguintes critérios:

1. Análise dos componentes principais (fatores com eigenvalue $\geq 1,0$ ).

2. Importância do fator (porcentagem mínima de $3 \%$ de variância explicada pelo componente).

3. Observação do scree plot.

4. Cargas fatoriais dos itens superiores a 0,30 (Pasquali, 1999). A análise fatorial de componente principal in- dicou oito fatores com eigenvalues acima de 1,0, sendo que cada um deles contribuía com, no mínimo, 4 \% da variância. Assim, pelos dois primeiros critérios poderiam ser extraídos oito fatores. A análise do gráfico scree plot (Figura 1) sugeriu, todavia, de forma mais clara que as soluções de três ou quatro fatores poderiam ser mais adequadas. Procedeu-se, então, à rotação Varimax para ambas as possibilidades, tendo-se utilizado como critério para retenção do item no fator a carga mínima de 0,30. 


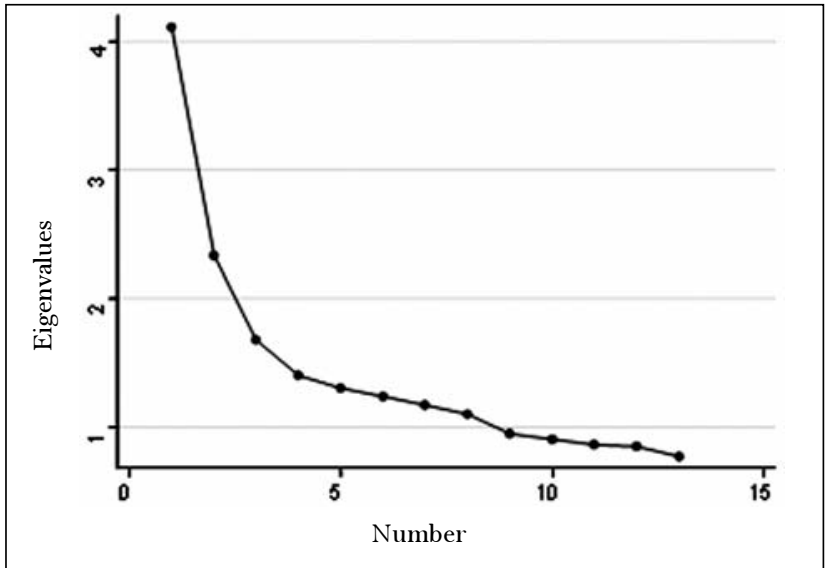

Geral

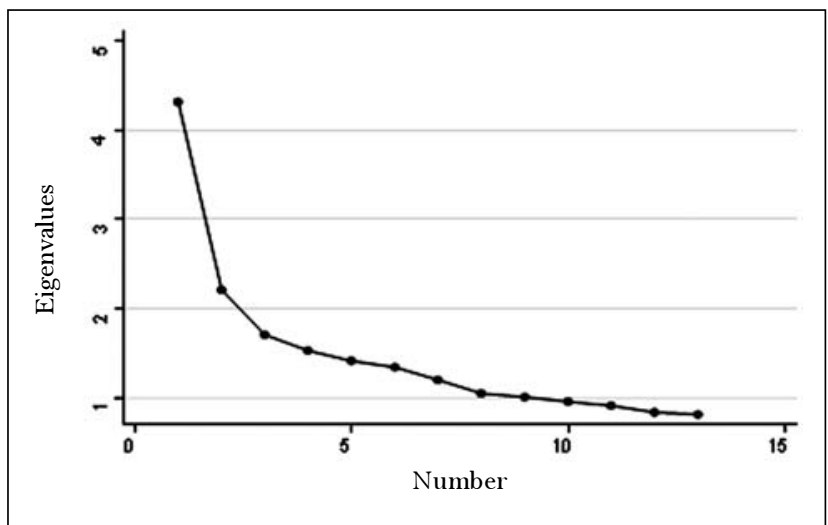

Feminino

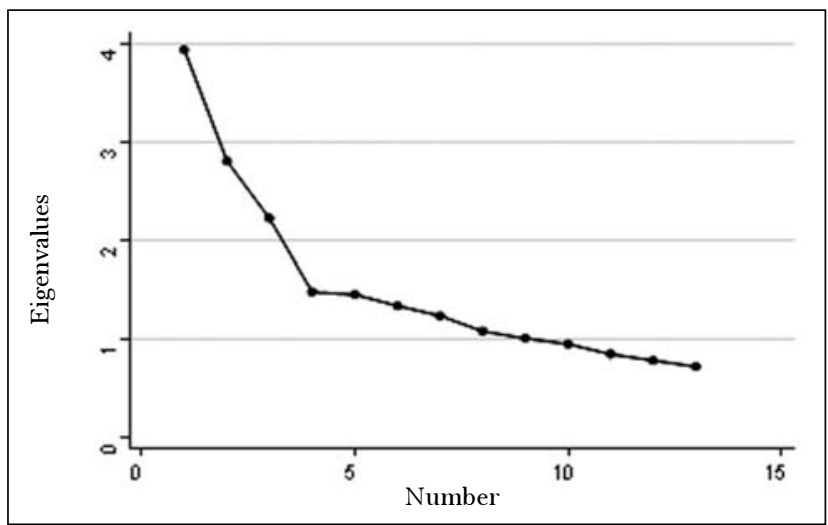

Masculino

Figura 1. Representação gráfica dos eigenvalues da análise fatorial, amostra geral e por sexo.

A solução de quatro fatores, mais semelhante à versão original da escala, mostrou-se inadequada porque o quarto fator ficaria constituído por apenas dois itens com conteúdos teóricos diferentes, motivo pelo qual se optou pela solução de três fatores (Tabela 3). O primeiro fator com eigenvalue 4,1 1, contribuiu com $16 \%$ da variância, o segundo fator com eigenvalue 2,33, 9\% da variância e o terceiro, com a eigenvalue 1,68 e 6\% da variância, totalizando $31 \%$ da variância.
Tabela 3

Cargas Fatoriais dos Itens na Solução Tri-Dimensional da Rotação Varimax

\begin{tabular}{|c|c|c|c|}
\hline \multirow[t]{2}{*}{ Item } & \multicolumn{3}{|c|}{ Fator } \\
\hline & f1 & $\mathrm{f} 2$ & f3 \\
\hline 2 & 0,47 & 2 & 0,47 \\
\hline 4 & 0,65 & 4 & 0,65 \\
\hline 8 & 0,47 & 8 & 0,47 \\
\hline 10 & 0,47 & 10 & 0,47 \\
\hline 14 & 0,52 & 14 & 0,52 \\
\hline 17 & 0,59 & 17 & 0,59 \\
\hline 19 & 0,37 & 19 & 0,37 \\
\hline 20 & 0,71 & 20 & 0,71 \\
\hline 22 & 0,44 & 22 & 0,44 \\
\hline 25 & 0,64 & 25 & 0,64 \\
\hline 26 & 0,59 & 26 & 0,59 \\
\hline $1^{*}$ & 0,17 & $1^{*}$ & 0,17 \\
\hline $11^{*}$ & $-0,06$ & $11^{*}$ & $-0,06$ \\
\hline $12^{*}$ & $-0,04$ & $12^{*}$ & $-0,04$ \\
\hline $13^{*}$ & 0,03 & $13^{*}$ & 0,03 \\
\hline $15^{*}$ & $-0,01$ & $15^{*}$ & $-0,01$ \\
\hline $21^{*}$ & $-0,06$ & $21^{*}$ & $-0,06$ \\
\hline $24^{*}$ & 0,11 & $24^{*}$ & 0,11 \\
\hline 3 & 0,22 & 3 & 0,22 \\
\hline $5^{*}$ & 0,11 & $5^{*}$ & 0,11 \\
\hline $6^{*}$ & $\mathrm{O}, 04$ & $6^{*}$ & 0,04 \\
\hline $16^{*}$ & 0,25 & $16^{*}$ & 0,25 \\
\hline 18 & 0,33 & 18 & 0,33 \\
\hline 23 & 0,34 & 23 & 0,34 \\
\hline 7 & 0,06 & 7 & 0,06 \\
\hline 9 & 0,21 & 9 & 0,21 \\
\hline
\end{tabular}

Nota. ${ }^{*}$ itens negativos.

Os itens $2,4,8,10,14,17,19,20,22,25$, e 26, são os que apresentaram maior carga no Fator 1, com eigenvalues entre 0,37 e 0,71. Do ponto de vista teórico, eles se encontram predominantemente associados à habilidade em identificar e descrever sentimentos e distingui-los de sensações corporais, uma das principais dimensões da alexitimia (cópia da escala pode ser solicitada à autora via e-mail). Sete itens apresentaram correlação positiva com o fator 2: 1, 11, 12, 13, 15, 21 e 24. Esses são itens negativos, isto é, expressam característica oposta às de alexitimia (Taylor et al., 1988). As cargas fatoriais foram altas, tendo oscilado entre 0,40 e 0,57 . Revelam um estilo de pensamento concreto, baseado na realidade, destituído de imaginação e fantasia, característico do chamado "pensamento operatório”.

O exame do fator 3 indica que os itens 3,5 e 16 tiveram carga negativa expressiva no fator, e insignificante nos demais. Quanto aos itens 6, 18 e 23, tiveram cargas suficientes $(>0,30)$ em dois fatores caracterizando-se como itens complexos. Estão relacionados a duas dimensões da versão original da TAS: os itens 5, 16 e 18 à "capacidade de fantasiar" (daydreaming) e os itens 3, 6 e 23 à "habilidade 
de expressar os sentimentos e as emoções". Ainda que os itens complexos devessem ser descartados por se associarem a mais de um fator, sugere-se a retenção do item 6 por se alinhar teoricamente com os demais, além de ter carga bem mais elevada no fator 3 do que no fator 2 (O,50 e 0,37 , respectivamente). Quanto aos itens 18 e 23 , devem ser descartados. Nesta medida, o fator 3 fica representado pelos itens 3, 5, 6 e 16. Esse fator assim constitui uma medida da habilidade de expressão de afetos e fantasias, prejudicadas em alexitímicos. Deve-se ainda mencionar que os itens 7 (Saber as respostas dos problemas é mais importante do que saber as razões das respostas), e 9 (Gosto de deixar as pessoas saberem minha opinião sobre as coisas) não apresentaram carga suficiente em nenhum dos fatores, precisando ser reformulados, ou mesmo descartados da escala, quando aplicada a populações com perfil semelhante ao da pesquisa atual.

\section{Discussão}

Os resultados sugerem que a versão em português da TAS com pacientes de hospital geral, apresenta índices satisfatórios de precisão, tanto de consistência interna, quanto de teste e reteste.

Quanto à estrutura fatorial, parece melhor representada por três fatores. O primeiro, indicativo da «dificuldade em discriminar e reconhecer os sentimentos e de discriminá-los de sensações corporais» manteve basicamente a composição de itens já verificada em outras pesquisas de validação da TAS que envolveram, inclusive, amostras com diferentes níveis sócio-econômico-cultural bastante diversos do estudo atual. Comparando-se, por exemplo, os resultados encontrados com os da amostra de universitários (Yoshida, 2000), verifica-se a compatibilidade destes itens com o F1 daquela pesquisa, em que, aparece também o item 1 . Há também equivalência satisfatória com o F1 da versão uruguaia da TAS, também com universitários (Rodrigo et al., 1989) e com o F1 a da versão original da escala, em inglês, com exceção dos itens 2 e 19, que pertencem respectivamente aos fatores 2 e 4 (Taylor et al., 1985). Considerando-se, portanto, a pertinência teórica dos itens e a robustez do fator, sugerese a retenção de todos os itens indicados anteriormente como pertencentes ao fator 1, quando se avaliar populações clínicas brasileiras.

O segundo fator ficou composto por itens que na versão original compõem predominantemente a dimensão F4, que avalia "a focalização em eventos externos e o controle das emoções" (itens em comum: 11, 13, 21 e 24) (Taylor et al., 1985). Na pesquisa com os universitários, estes itens compuseram o F3, ao lado dos itens 7 e 19. O item 1 apareceu no F1 e os itens 12 e 15 tiveram carga em F4. Estas diferenças não chegam a comprometer a interpretabilidade do fator, motivo pelo qual recomenda-se que, para populações clínicas, os itens identificados sejam retidos. Por outro lado, seria desejável a adaptação ou o desenvolvimento de novos itens, de modo a que o "pólo positivo" da alexitimia também pudesse ser contemplado, uma vez que este fator ficou composto exclusivamente por itens negativos. O intuito seria o de torná-lo mais "equilibrado" no que concerne à representatividade da dimensão medida.

E o terceiro, apesar de composto por itens de duas dimensões da versão original - "dificuldade em descrever sentimentos" (F2) e "reduzida capacidade de fantasiar (daydreaming)" (F3), parece medir a dificuldade de expressão de afetos e de fantasias, sendo portanto razoável que ele seja mantido para a avaliação de pacientes de hospital geral. Além disto, devem ser excluídos os itens 7 e 9 , por não terem cargas expressivas em nenhum dos três fatores. Com isto a versão da TAS para população clínica brasileira fica reduzida a 22 itens.

A solução de três fatores coincide parcialmente com a encontrada por Haviland et al. (1988) em amostra de pacientes internados por abuso de substâncias, composta por um fator 1- que mede a habilidade de identificar sentimentos e distinguí-los de sensações corporais, fator 2- sonhar acordado, e fator 3 - pensamento externamente orientado. Por outro lado, ela contrasta com a solução de quatro fatores, verificada junto a estudantes brasileiros e também em diferentes culturas e patologias, conforme referido na introdução. Em todos os casos, no entanto, a estrutura do fator 1 mantém-se muito semelhante, sugerindo especialmente a validade desta dimensão. Este fato, aponta na direção do defendido por Haviland et al. (1988) de que "a TAS não deve ser utilizada como uma única medida para avaliar alexitimia . . . devendo-se dividi-la em três ou quatro sub-escalas" (p. 84). E neste sentido, cotejar em pesquisas futuras, a TAS com outras medidas de alexitimia, tais como a TAS-2O ou ainda a OAS pode ser relevante para o processo de validação destas escalas, permitindo que se tenha maior clareza das propriedades psicométricas de cada uma delas entre nós.

\section{Referências}

Bagby, R. M., Parker, J. D., \& Taylor, G. J. (1994). The twentyitem Toronto Alexithymia Scale I. Item selection and crossvalidation of the factor structure. Journal of Psychosomatic Research, 38, 23-32.

Bagby, R. M., Taylor, G. J., \& Atkinson, L. (1988). Alexithymia: A comparative study of three self-report measures. Journal of Psychosomatic Research, 32, 107-116.

Bagby, R. M., Taylor, G. J., \& Parker, J. D. (1994). The twentyitem Toronto Alexithymia Scale II. Convergent, discriminant, and concurrent validity. Journal of Psychosomatic Research, 38, $33-40$.

Bagby, R. M., Taylor, G. J., \& Parker, J. D. A. (2003). Nomological domain of the alexithymia construct. Retirado de http:// spitswww.uvt.nl/web/fsw/psychologie/ emotions2003/2/ h9.pdf.

Bagby, R. M., Taylor, G. J., Parker, J. D. A., \& Loiselles, C. (1990). Cross-validation of the factor structure of the Toronto Alexithymia Scale. Journal of Psychosomatic Research, 34, 4751.

Bagby, R. M., Taylor, G. J., \& Ryan, D. (1986). Toronto Alexithymia Scale: Relationship with personality and psychopathology measures. Psychotherapy and Psychosomatics, 45, 207-215. 
Bressi, C., Taylor, G. J., Parker, J. D., Bressi, S., Brambilla, V., \& Aguglia, E. et al. (1996). Cross-validation of the factor structure of the 20-Item Toronto Alexithymia Scale: An Italian multicenter study. Journal of Psychosomatic Research, $41,551-559$

Campbell, R. J. (1996). Psychiatric Dictionary (7. ed.). New York: Oxford University Press.

Carneiro, B. V. (2006). Escala de Alexitimia pelo Observador (OAS), precisão e validade da versão em português. Projeto de pesquisa para Tese de Doutorado não-publicado, Pós Graduação em Psicologia, Pontifícia Universidade Católica de Campinas, SP.

Consoli, S. M. (1996). Les approches disposicionnelles en psychologie de la santé. Psychologie Française, 4112), 117-130.

Freyberger, H. (1977). Supportive psychotherapeutic techniques in primary and secundary alexithymia. Psychotherapy $E^{\circ}$ Psychosomatics, 28, 337-342.

Fukunishi, I., Kikuchi, M., Kaji, N., \& Yamasaki, K. (1997). Can scores on alexithymia distinguish patients with peptic ulcer and erosive gastritis? Psychological Reports, 80, 995-1004.

Haviland, M. G. (1998). The validity of the California Q-set alexithymia prototype. Psychosomatics, 39, 536-539.

Haviland, M. G., \& Reise, S. P (1996a). A California Q-set Alexithymia Prototype and its relationship to ego-control and ego-resiliency. Journal of Psychosomatic Research, 41, 597-608.

Haviland, M. G., \& Reise, S. P. (1996b). Structure of the TwentyItem Toronto Alexithymia Scale. Journal of Personality Assessment, 66(1), 116-125.

Haviland, M. G., Shaw, D. G., MacMurray, J. P., \& Cummings, M. A. (1988). Validation of the Toronto Scale with substance abusers. Psychotherapy \& Psychosomatics, 50, 81-87.

Haviland, M. G., Warren, A. L., \& Riggs, M. L. (2000). An Observer Scale to measure alexithymia. Psychosomatics, 41(5), 385-392.

Haviland, M. G., Warren, A. L., Riggs, M. L., \& Gallacher, M. (2001). Psychometric properties of the Observer Alexithymia Scale in a clinical sample. Journal of Personality Assessment, 77(1), 176-186.

Haviland, M. G., Warren, A. L., Riggs, M. L., \& Nitch, S. R. (2002). Concurrent validity of two observer-rated alexithymia measures. Psychosomatics, 43(6), 472-477.

Jacob, S., \& Hautekeete, M. (1998). Alexithymie et mémoire: un critère d'acceptation plus rigoureux dans les tâches de reconnaissance. L'Éncéphale, 24, 199-477.

Kauhanen, J., Julkunen, J., \& Salonen, J. T. (1991). Alexithymia and perceived symptoms: Criterion validity of the Toronto Alexithymia Scale. Psychotherapy and Psychosomatics, 56, 247252.

Kosturek, A., Gregory, R. J., Sousou, A. J., \& Trief, P. (1998). Alexithymia and somatic amplification in chronic pain. Psychosomatics, 39, 399-404.

Larsen, J. K., Brand, N., Bermond, B., \& Hijman, R. (2003). Cognitive and emotional characteristics of alexithymia: A review of neurobiological studies. Journal of Psychosomatic Research, 54, 533-541.

Maciel, M. J. N., \& Yoshida, E. M. P. (2006). Avaliação da alexitimia, neuroticismo e depressão em dependentes de álcool. Avaliação Psicológica, 5(1), 43-54.

Madioni, F., \& Mammana, L. A. (2001). Toronto Alexithymia Scale in outpatients with sexual disorders. Psychopathology, 34, 95-98.

Martinez-Sánchez, F., A to-García, M., \& Ortiz-Soria, B. (2003). Alexithymia-state or trait? The Spanish Journal of Psychology, $6(1), 51-59$.
Marty, P., \& M'Uzan, M. (1994). O pensamento operatório. Revista Brasileira de Psicanálise, 28(1), 165-174. (Original publicado em 1963)

Medeiros, L. P. (2002). Eficácia adaptativa e grau de alexitimia em pacientes com Doença de Chron e Retocolite Ulcerativa Inespecífica. Dissertação de Mestrado não-publicada, Pós Graduação em Psicologia, Pontifícia Univer sidade Católica de Campinas, SP.

Millard, R. W., \& Kinsler, B. L. (1992). Evaluation of constricted affect in chronic pain: An attempt using the Toronto Alexithymia Scale. Pain, 50, 287-192.

Parker, J. D., Bagby, R. M., \& Taylor, G. J. (1991). Alexithymia and depression: Distinct or overlapping constructs? Comprehensive Psychiatry, 32, 387-394.

Pasquali, L. (Ed.). (1999). Instrumentos psicológicos: Manual prático de elaboração. Brasília, DF: LabPA/IBAPP.

Pedinielli, J. L., \& Rouan, G. (1998). Concept d'alexithymie et son intérêt en psychosomatique. Encyclopédie MédicoChirurgicale, 37(400), D-20.

Pregnolatto, A. P. F. (2005). Alexitimia e sintomas psicopatológicos em pacientes submetidos a hemodiálise. Dissertação de Mestrado não-publicada, Pós Graduação em Psicologia, Pontifícia Universidade Católica de Campinas, SP.

Rodrigo, G., Lusiardo, M., \& Normey, L. (1989). Alexithymia: Reliability and validity of the spanish version of the Toronto Alexithymia Scale. Psychotherapy and Psychosomatics, 51, 162168.

Sifneos, P. (1977). Psychothérapie brève et crise émotionnelle (G. Hougardy \& D. Luminet, Trads.). Bruxelles, Belgique: Pierre Mardaga. (Original publicado em 1972)

Sifneos, P. E., Apfel-Savitz, R., \& Frankel, F. H. (1977). Alexithymia: Observations in psychosomatic patients. Psychotherapy and Psychosomatics, 28, 47-57.

Taylor, G. J., Bagby, R. M. \& Luminet, O. (2000). Assessment of alexithymia:self-report and observer-rated measures. In J. D. A. Parker \& R. Bar-On (Eds.), The handbook of emotional intelligence (pp. 301-319). São Francisco, CA: Jossey Bass.

Taylor, G. J., Bagby, R. M., Ryan, D. P., Parker, J. D., Doody, K. F., \& Keefe, P. (1988). Criterion validity of the Toronto Alexithymia Scale. Psychosomatic Medicine, 50, 500-509.

Taylor, G. J., Ryan, D., \& Bagby, R. M. (1985). Toward the development of a new self-report alexithymia scale. Psychotherapy and Psychosomatics, 44, 191-199.

Wiethaeuper, D., Balbinotti, M. A. A., Pelisoli, C., \& Barbosa, M. L. L. (2005). Estudos da consistência interna e fatorial confirmatória da ETA-20 com estudantes brasileiros. Revista Interamericana de Psicologia, 39(2), 1-17.

Wise, T. N., Mann, L. S., \& Randell, P. (1995). The stability of alexithymia in depressed patients. Psychopathology, 28, 173176.

Wise, T. N., Osbourne, C., Strand, J., Fagan, P. J., \& Schmidt, C. W. J. R. (2002). Alexithymia in patients attending a sexual disorders clinic. Journal of Sex and Marital Therapy, 28(5), 445450 .

Yoshida, E. M. P. (2000). Toronto Alexthymia Scale - TAS: Precisão e validade da versão em português. Psicologia: Teoria e Prática, 2(1), 59-74.
Recebido: 23/11/2005 $1^{a}$ revisão: $29 / 05 / 2006$ $2^{a}$ revisão: $11 / 12 / 2006$ Aceite final: 19/12/2006 